\title{
Coproximinality in the Space of Bounded Functions
}

\author{
Eyad Abu-Sirhan and Zuhier Altawallbeh \\ Department of Mathematics, Tafila Technical University, Tafila 6610, Jordan \\ Correspondence should be addressed to Zuhier Altawallbeh; tawalbh@nmsu.edu \\ Received 16 January 2014; Accepted 25 March 2014; Published 7 April 2014 \\ Academic Editor: Ram N. Mohapatra
}

Copyright (C) 2014 E. Abu-Sirhan and Z. Altawallbeh. This is an open access article distributed under the Creative Commons Attribution License, which permits unrestricted use, distribution, and reproduction in any medium, provided the original work is properly cited.

As a counterpart to best approximation in Banach spaces, the best coapproximation was introduced by Franchetti and Furi (1972). In this paper, we shall consider the relation between coproximinality of a nonempty subset $M$ of a Banach space $X$ and of $C(S, M)$ in $C(S, X)$.

\section{Introduction}

Let $M$ be a nonempty subset of a Banach space $X$. We say that $M$ is proximinal in $X$ if each $x \in X$ there corresponds at least to one point $m_{0} \in M$ such that

$$
\left\|x-m_{0}\right\| \leq\|x-m\|, \quad \forall m \in M .
$$

Recently, another kind of approximation from $M$ has been introduced by Franchetti and Furi [1] who have considered those elements (if any) $m_{0} \in M$ satisfying

$\left\|m-m_{0}\right\| \leq\|x-m\|, \quad \forall m \in M$, and for any fixed $x \in X$.

Such an element $m_{0}$ is called best coapproximant of $x$ in $M$. The set of all such elements, satisfying above inequality, is denoted by $R_{M}(x)$. The subset $M$ is called coproximinal in $X$ if $R_{M}(x)$ is nonempty for any $x \in X$. If $R_{M}(x)$ is singleton for any $x \in X$, then $M$ is called coChebyshev; see [2-4].

It is clear that $R_{M}(x)$ is convex if $M$ is convex and closed. The kernal of $R_{M}$ is the set defined by

$$
\operatorname{ker} R_{M}=\{x \in X:\|m\| \leq\|m-x\|, \forall m \in M\} .
$$

Many results in the theory of best coapproximation have appeared since Franchetti and Furri's paper, 1972. These results are largely concerned with the question of existence and uniqueness of best coapproximation (see for example [5$8])$. Let $\ell_{\infty}(S, X)$ and $C(S, X)$ denote the Banach spaces of all bounded (resp., continuous) functions from a topological space $S$ into a Banach space $X$ and let $M$ be a closed subset of $X$. It should be remarked that if $S$ is a compact space, then $C(S, X)$ is a subspace of $\ell_{\infty}(S, X)$. In this paper, we discuss the coproximinality of $\ell_{\infty}(S, M)$ and $C(S, M)$ in $\ell_{\infty}(S, X)$ and $C(S, X)$, respectively. For uniqueness and existence of best coapproximation in $C(S, X)$, see [7].

Definition 1. Let $M$ be a coproximinal subset of a Banach space $X$. A map $F_{M}: X \rightarrow M$ which associates with each element in $X$ one of its best coapproximation in $M$ (i.e., $F_{M}(x) \in R_{M}(x)$ for all $\left.x \in X\right)$ is called a coproximity map.

Remark 2. Let $M$ be a coproximinal subset of a Banach space $X$. We state some basic properties of a proximity map $F_{M}$.

(1) If $M$ is coChebyshev, [5], then

(a) $F_{M}(m)=m$, for any $m \in M$.

(b) $F_{M}(\alpha x)=\alpha F_{M}(x)$, for any scalar $\alpha$ and $x \in X$. (i.e., $F_{M}$ is homogeneous).

(c) $F_{M}(m+x)=m+F_{M}(x)$, for any $x \in X$ and $m \in M$.

(2) If $M$ is a subspace of $X$, then

(a) $\left\|F_{M}(x)-F_{M}(y)\right\| \leq\|x-m\|+\|y-m\|$, for any $x, y \in X$ and $m \in M$.

(b) $\left\|F_{M}(x)\right\| \leq\|x\|$, for all $x \in X$. (set $y=0$ and $m=$ 0 in (a) and take into account that $F_{M}(0)=0$ ). 
(c) $F_{M}$ is continuous at $x=0$.

(d) If $F_{M}$ is linear, then $F_{M}$ is continuous.

Theorem 3 (see [3]). If $M$ is coproximinal hyperplane or 1dimensional subspace of a Banach space $X$, then $M$ has a continuous coproximity map.

Lemma 4 (see [3]). Let $M$ be a coproximinal subspace of $X$. Then the following are equivalent.

(1) $M$ has linear coproximity map.

(2) $\operatorname{ker} R_{M}$ contains a closed subspace $W$ such that $X=$ $W \oplus M$. Moreover, if (2) holds, then the linear coproximity map for $M$ can be defined by $P(m+w)=m$, $m \in M$, and $w \in W$.

Definition 5. Let $X$ be a Banach space. Consider the following.

(a) A subspace $M$ of $X$ is called one complemented in $X$ if there is a closed subspace $W$ such that $X=W \oplus M$ and the projection $P: X \rightarrow M$ is contractive.

(b) A linear projection $P$ is called an $M$-projection if $\|x\|=\max \{\|P(x)\|,\|x-P(x)\|\}$ for all $x \in X$. A closed subspace $J$ of $X$ is called an $M$-summand if it is the range of an $M$-projection, [9].

(c) A linear projection $P$ is called an $L$-projection if $\|x\|=$ $\|P(x)\|+\|x-P(x)\|$ for all $x \in X$. A closed subspace $J$ of $X$ is called an $L$-summand if it is the range of an $L$-projection.

Clearly every $M$-summand is one complemented.

Theorem 6. Let $M$ be a subspace of a Banach space $X$. Then the following are equivalent.

(1) $M$ is one complemented in $X$.

(2) $M$ is coproximinal in $X$ and has linear coproximity map.

Proof. (1) $\rightarrow$ (2): Let $X=M \oplus W, P: X \rightarrow M$ a contractive projection, and $x \in X$. Then $x=m_{1}+w_{1}$,

$$
\begin{gathered}
\|P(x-m)\| \leq\|x-m\|, \quad \forall m \in M, \\
\left\|m-m_{1}\right\| \leq\|x-m\|, \quad \forall m \in M .
\end{gathered}
$$

Hence $M$ is coproximinal in $X$. Now let $w \in W$. Then for any $m \in M,\|P(w-m)\|=\|m\| \leq\|w-m\|$. Thus $W \subset \operatorname{ker} R_{M}$ and $M$ has linear coproximity map by Lemma 4 .

$(2) \rightarrow$ (1): If $M$ has a linear coproximity map, then ker $R_{M}$ contains a closed subspace $W$ such that $X=M \oplus W$. Moreover, $P(m+w)=m, m+w \in M \oplus W$ is a linear coproximity map for $M$ and so $P$ is the projection of $M$ along $W$ by Lemma 4 . To show that $P$ is contractive, if $x \in X$, then $\|m-P(x)\| \leq\|m-x\|$ for all $m \in M$, and so $\|p(x)\| \leq\|x\|$ which means that $P$ is contractive.

Corollary 7. If $M$ is $L$-summand, then $M$ is coproximinal and has continuous coproximity map.
For more information about coproximinal sets, optimal sets, and contractive sets, the reader is referred to [10-18].

\section{Coproximinality in $\ell_{\infty}(S, X)$ and $C(S, X)$}

Let $S$ be a compact Hausdorff space and $X$ be a Banach space. We denote $\ell_{\infty}(S, X)$ and $C(S, X)$ to be the Banach space of all bounded ( resp., continuous) functions from $S$ into $X$.

Lemma 8 (see [19]). Let A be a continuous map of a Banach space $X$ into a closed subset $Y$ of $X$. If $S$ is compact Hausdorff space, then the map defined by $\Phi(z)=$ Aoz is continuous from $C(S, X)$ to $C(S, Y)$.

Lemma 9. Let $M$ be a closed subset of a Banach space $X$. If there is a continuous coproximity map of $X$ onto $M$, then $C(S, M)$ is coproximinal in $C(S, X)$ and in fact it has a continuous coproximity map.

Proof. Let $\phi: X \rightarrow M$ be a continuous coproximity map for $M$. Define

$$
\Phi: C(S, X) \longrightarrow C(S, M)
$$

by $\Phi(f)=\phi \circ f$, then $\Phi$ is continuous by Lemma 8 . Let $h \in$ $C(S, M)$, then

$$
\|h(s)-\phi(f(s))\| \leq\|f(s)-h(s)\|
$$

for all $s \in S$,

$$
\|h-\phi o f\| \leq\|f-h\|
$$

Hence

$$
\|h-\Phi(f)\| \leq\|f-h\|
$$

for all $h \in C(S, M)$ and $\Phi$ is a continuous coproximity map from $C(S, X)$ onto $C(S, M)$.

Lemma 10. Let $M$ be a closed subset of a Banach space $X$. If $C(S, M)$ is coproximinal in $C(S, X)$, then $M$ is coproximinal in $X$. Moreover, if $C(S, M)$ has a continuous coproximity map, then $M$ has a continuous coproximity map.

Proof. For $x \in X$, define $f_{x}: S \rightarrow X$ by $f_{x}(s)=x$ for all $s \in S$. The map $f_{x} \in C(S, X)$. By assumption, there exists $g_{0} \in C(S, M)$ such that

$$
\left\|g_{0}-h\right\| \leq\left\|f_{x}-h\right\|
$$

for all $h \in C(S, M)$. In particular,

$$
\left\|g_{0}-f_{m}\right\| \leq\left\|f_{x}-f_{m}\right\|
$$

for all $m \in M$. For any fixed $s_{0} \in S$,

$$
\left\|g_{0}\left(s_{0}\right)-m\right\| \leq\|x-m\|
$$

for all $m \in M$ and so $g_{0}\left(s_{0}\right)$ is a best coapproximation of $x$. Now let $\Phi: C(S, X) \rightarrow C(S, M)$ be a continuous coproximity map. For any fixed $s_{0} \in S$, define $\phi: X \rightarrow M$ 
by $\phi(x)=\Phi f_{x}\left(s_{0}\right)$. Then $\phi$ is a proximity map. Suppose that $x_{n} \rightarrow x$ in $X$. Then $\Phi\left(f_{x_{n}}\right) \rightarrow \Phi\left(f_{x}\right)$. and

$$
\begin{aligned}
\left\|\phi\left(x_{n}\right)-\phi(x)\right\| & =\left\|\Phi\left(f_{x_{n}}\right)\left(s_{0}\right) \longrightarrow \Phi\left(f_{x}\right)\left(s_{0}\right)\right\| \\
& \leq\left\|\Phi\left(f_{x_{n}}\right)-\Phi\left(f_{x}\right)\right\| \longrightarrow 0
\end{aligned}
$$

Hence $\phi$ is continuous.

Theorem 11. Let $M$ be a closed subset of a Banach space X. Then the following are equivalent.

(1) $M$ is coproximinal in $X$ and has a continuous coproximity map.

(2) $C(S, M)$ is coproximinal in $C(S, X)$ and has a continuous coproximity map.

Proof. The proof follows from Lemmas 9 and 10.

Theorem 12. Let $M$ be a closed subset of a Banach space $X$ and let $S$ be a topological space. Then the following are equivalent.

(1) $M$ is coproximinal in $X$.

(2) $\ell_{\infty}(S, M)$ is coproximinal in $\ell_{\infty}(S, X)$.

Proof. (1) $\rightarrow$ (2). Let $f \in \ell^{\infty}(S, X)$. Since $M$ is coproximinal in $X$, then $R_{M}(f(s))$ is nonempty for any $s \in S$. By axiom of choice, we may define a function $h: S \rightarrow M$ such that $h(s) \in R_{M}(f(s))$ for all $s \in S$. Since $\|h(s)\| \leq\|f(s)\|$ for all $s \in S, h \in \ell_{\infty}(S, M)$ and $h$ is a best coapproximation of $f$ in $\ell_{\infty}(S, X)$.

$(2) \rightarrow$ (1). For $x \in X$, define $f_{x}: S \rightarrow X$ by $f_{x}(s)=x$ for all $s \in S$. Since $\ell_{\infty}(S, M)$ is coproximinal in $\ell_{\infty}(S, X)$, the map $f_{x} \in \ell_{\infty}(S, X)$. By assumption, there exists $g_{0} \in \ell_{\infty}(S, M)$ such that

$$
\left\|g_{0}-h\right\| \leq\left\|f_{x}-h\right\|
$$

for all $h \in \ell_{\infty}(S, M)$. In particular,

$$
\left\|g_{0}-f_{m}\right\| \leq\left\|f_{x}-f_{m}\right\|
$$

for all $m \in M$. For any fixed $s_{0} \in S$,

$$
\left\|g_{0}\left(s_{0}\right)-m\right\| \leq\|x-m\|
$$

for all $m \in M$ and $g_{0}\left(s_{0}\right)$ is a best coapproximation of $x$.

We recall that.

Definition 13. Let $\Phi$ be a set valued mapping, taking points of a topological space $S$ into the family of all subsets of a topological space $T$. The mapping $\Phi$ is said to be lower semicontinuous if, for each open set $O$ in $T$, the set $\Phi^{-}=\{s \in$ $S: \Phi(s) \cap O \neq \phi\}$ is open.

Theorem 14 (see [19, Michael Selection Theorem]). Let $\Phi$ be a lower semicontinuous of a paracompact space $S$ into the family of nonvoid closed convex subsets of a Banach space $X$. Then $\Phi$ has a continuous selection; that is, there exists a continuous map $\varphi: S \rightarrow X$ such that $\varphi(s) \in \Phi(s)$ for all $s \in S$.
Definition 15. A sequence of sets $\left\{A_{n}\right\}$ in a topological space $(X, T)$ is convergent and has the limit $A$ if and only if $\lim \sup \left\{A_{n}\right\}=A=\lim \inf \left\{A_{n}\right\}$ where $\lim \sup \left\{A_{n}\right\}$ is the set of those elements which are limits of points in $A_{n}$ for infinitely many $n$ and $\lim \inf \left\{A_{n}\right\}$ is the set of those elements which are limits of points in $A_{n}$ for cofinitely many (for all but finitely many) $n$.

Lemma 16. Let $S$ be a compact Hausdorff space, $X$ a Banach space, and $G$ a compact subset of $X$. For $f \in C(S, X)$, we define $\pi_{f}: S \rightarrow 2^{G}$ by

$$
\pi_{f}(s)=\left\{g_{0} \in G:\left\|g_{0}-g\right\| \leq\|g-f(s)\|, \forall g \in G\right\} .
$$

Then $\pi_{f}$ is lower semicontinuous.

Proof. Let $O$ be an open set in $G$. We need to prove that

$$
\pi_{f}^{-}(O)=\left\{s \in S: \pi_{f}(s) \cap O \neq \phi\right\}
$$

is open. For this purpose, let $\left\{x_{n}\right\}$ be a sequence in $S / \pi_{f}^{-}(O)$ and $x_{n} \rightarrow x$ as $n \rightarrow \infty$, so $x_{n} \notin \pi_{f}^{-}(O)$ for all $n$ which means that

$$
\pi_{f}\left(x_{n}\right) \cap O=\phi .
$$

Thus $\pi_{f}\left(x_{n}\right) \subset G / O$ for all $n$, and $\left\{\pi_{f}\left(x_{n}\right)\right\}$ is a sequence of subsets of $G$. We shall show that $\pi_{f}\left(x_{n}\right) \rightarrow \pi_{f}(x)$ as $n \rightarrow \infty$. First, we show that

$$
\lim \sup \pi_{f}\left(x_{n}\right) \subseteq \pi_{f}(x) .
$$

Let $z \in \lim \sup \pi_{f}\left(x_{n}\right)$. Then there is a sequence of points $\left\{z_{k}\right\}$ and a subsequence $\left\{\pi_{f}\left(x_{n_{k}}\right)\right\}$ of $\left\{\pi_{f}\left(x_{n}\right)\right\}$ such that $z_{k} \in$ $\pi_{f}\left(x_{n_{k}}\right)$ and $z_{k} \rightarrow z$ as $k \rightarrow \infty$. By the definition of $\pi_{f}$, we get

$$
\left\|z_{k}-g\right\| \leq\left\|f\left(x_{n_{k}}\right)-g\right\| \quad \forall g \in G .
$$

Taking $k \rightarrow \infty$ and $n_{k} \rightarrow \infty$, and since $f$ is continuous, we have

$$
\|z-g\| \leq\|f(x)-g\| \quad \forall g \in G,
$$

so $z \in \pi_{f}(x)$. This proves that $\lim \sup \pi_{f}\left(x_{n}\right) \subseteq \pi_{f}(x)$. Now we shall show that $\pi_{f}(x) \subseteq \liminf \pi_{f}\left(x_{n}\right)$. Let $z \notin$ $\lim \inf \pi_{f}\left(x_{n}\right)$. In this case, if $z_{k} \rightarrow z$ as $k \rightarrow \infty$ then $z_{k} \notin \pi_{f}\left(x_{k}\right)$ for infinitely many $k$, and, so,

$$
\left\|z_{k}-g_{k}\right\|>\left\|f\left(x_{k}\right)-g_{k}\right\| \quad \text { for some } g_{k} \in G \text {. }
$$

We may assume that, without loss of generality, $g_{k} \rightarrow g$ as $k \rightarrow \infty$, for some $g \in G$, since $G$ is compact. Taking $k \rightarrow$ $\infty$, we have that

$$
\|z-g\|>\|f(x)-g\|
$$

so $z \notin \pi_{f}(x)$ and we have as a conclusion the follwing result:

$$
\begin{aligned}
\liminf \pi_{f}\left(x_{n}\right) \subseteq \lim \sup \pi_{f}\left(x_{n}\right) \subseteq \pi_{f}(x) \\
\subseteq \liminf \pi_{f}\left(x_{n}\right) .
\end{aligned}
$$


Thus, $\lim \pi_{f}\left(x_{n}\right)=\pi_{f}(x)$. Finally, we know that $G / O$ is a closed set and the members of the sequence of sets $\left\{\pi_{f}\left(x_{n}\right)\right\}$ are all subsets of $G / O$ for all $n$. Now, to complete the proof, we still need to show that

$$
\lim \pi_{f}\left(x_{n}\right) \subseteq \frac{G}{O}
$$

To do so, let $z \in \limsup \pi_{f}\left(x_{n}\right)$. By the definition of $\limsup \pi_{f}\left(x_{n}\right)$, there is a sequence of points $\left\{z_{k}\right\}$ and a subsequence $\left\{\pi_{f}\left(x_{n_{k}}\right)\right\}$ of the sequence $\left\{\pi_{f}\left(x_{n}\right)\right\}$ such that

$$
\begin{gathered}
z_{k} \in \pi_{f}\left(x_{n_{k}}\right) \subseteq \frac{G}{O} \quad \forall k, \\
z_{k} \longrightarrow z \text { as } k \longrightarrow \infty .
\end{gathered}
$$

Since $G / O$ is closed, we get $z \in G / O$. This proves that $\pi_{f}(x) \subseteq$ $G / O$. This means that $\pi_{f}(x) \cap O=\phi$, and so $x \notin \pi_{f}^{-}(O)$. Thus $\pi_{f}^{-}(O)$ is open set and so $\pi_{f}$ is lower semicontinuous.

Theorem 17. Let $M$ be a compact convex subset of a Banach space $X$ and $S$ be a compact Hausdorffspace. Then the following are equivalent.

(1) $M$ is coproximinal in $X$.

(2) $C(S, M)$ is coproximinal in $C(S, X)$.

Proof. $(2) \rightarrow$ (1). The proof is similar to that given in Lemma 10.

$(1) \rightarrow(2)$. Let $f \in C(S, X)$ and $\pi_{f}: S \rightarrow 2^{M}$ as defined in Lemma 16. Then $\pi_{f}$ is lower semicontinuous by Lemma 16. Since $M$ is closed, nonvoid, and convex, then $\pi_{f}(s)$ is closed, nonvoid, and convex. By Michael Selection Theorem, $\pi_{f}$ has a continuous selection $g: S \rightarrow M$. Then $g \in C(S, M)$ and

$$
\begin{gathered}
\|g(s)-h(s)\| \leq\|f(s)-h(s)\|, \quad \forall s \in S, h \in C(S, M), \\
\|g-h\| \leq\|f-h\|, \quad \forall h \in C(S, M) .
\end{gathered}
$$

Hence $g$ is a best coapproximation of $f$ in $C(S, M)$.

Corollary 18. Let $S$ be a compact Hausdorff space and let $X$ be a reflexive Banach space. Then $B_{1}(X)$ (the closed unit ball of $X$ ) is coproximinal in $X$ if and only if $C\left(S, B_{1}(X)\right)$ is coproximinal in $C(S, X)$.

Proof. Since $X$ is reflexive, then $B_{1}(X)$ is compact by Alaoglu Theorem. As $B_{1}(X)$ is closed and convex subset of $X$, the result follows from Theorem 17.

Problem 19. If $S$ is a compact Hausdorff space and $X$ is a Banach space, when $C(S, X)$ is coproximinal in $\ell_{\infty}(S, X)$.

\section{Conflict of Interests}

The authors declare that there is no conflict of interests regarding the publication of this paper.

\section{Acknowledgment}

Finally the author would like to thank the referees for their valuable advice.

\section{References}

[1] C. Franchetti and M. Furi, "Some characteristic properties of real Hilbert spaces," Revue Roumaine de Mathématiques Pures et Appliquées, vol. 17, pp. 1045-1048, 1972.

[2] T. D. Narang, "On best coapproximation in normed linear spaces," The Rocky Mountain Journal of Mathematics, vol. 22, no. 1, pp. 265-287, 1992.

[3] W. H. Song, "A remark on coapproximation," Journal of Mathematical Research and Exposition, vol. 14, no. 4, pp. 547-551, 1994.

[4] D. C. Soni and L. Bahadur, "A note on coapproximation," Indian Journal of Pure and Applied Mathematics, vol. 22, no. 7, pp. 579$582,1991$.

[5] P. L. Papini and I. Singer, "Best coapproximation in normed linear spaces," Monatshefte für Mathematik, vol. 88, no. 1, pp. $27-44,1979$.

[6] G. S. Rao and K. R. Chandrasekaran, "Characterizations of elements of best coapproximation in normed linear spaces," Pure and Applied Mathematika Sciences, vol. 26, no. 1-2, pp. 139147, 1987.

[7] G. S. Rao and R. Saravanan, "Some results concerning best uniform coapproximation," Journal of Inequalities in Pure and Applied Mathematics, vol. 3, no. 2, article 24, 2002.

[8] G. S. Rao and R. Saravanan, "Strongly unique best coapproximation," Kyungpook Mathematical Journal, vol. 43, no. 4, pp. 519-538, 2003.

[9] E. Abu-Sirhan, "Best simultaneous approximation in function and operator spaces," Turkish Journal of Mathematics, vol. 36, no. 1, pp. 101-112, 2012.

[10] B. Beauzamy, "Projections contractantes dans les espaces de Banach," Bulletin des Sciences Mathématiques. 2e Série, vol. 102, no. 1, pp. 43-47, 1978.

[11] B. Beauzamy and B. Maurey, "Points minimaux et ensembles optimaux dans les espaces de Banach," vol. 24, no. 2, pp. 107139, 1977.

[12] R. E. Bruck, Jr., "Properties of fixed-point sets of nonexpansive mappings in Banach spaces," Transactions of the American Mathematical Society, vol. 179, pp. 251-262, 1973.

[13] R. E. Bruck, Jr., "Nonexpansive projections on subsets of Banach spaces," Pacific Journal of Mathematics, vol. 47, pp. 341-355, 1973.

[14] V. Davis and P. Eno, "Contractive projections on $l_{p}$-spaces," London Mathematical Society Lecture Note Series, vol. 137, pp. 151-161, 1989.

[15] D. G. de Figueiredo and L. A. Karlovitz, "On the extensions of contractions of normed spaces," in Proceedings of Symposia in Pure Mathematics, Nonlinear Functional Analysis, vol. 18, pp. 95-104, American Mathematical Society, 1970.

[16] L. Hetzelt, "On suns and cosuns in finite-dimensional normed real vector spaces," Acta Mathematica Hungarica, vol. 45, no. 1-2, pp. 53-68, 1985.

[17] A. Kamińska and G. Lewicki, "Contractive and optimal sets in modular spaces," Mathematische Nachrichten, vol. 268, pp. 7495, 2004. 
[18] G. Lewicki and G. Trombetta, "Optimal and one-complemented subspaces," Monatshefte für Mathematik, vol. 153, no. 2, pp. 115$132,2008$.

[19] W. A. Light and E. W. Cheney, Approximation Theory in Tensor Product Spaces, vol. 1169 of Lecture Notes in Mathematics, Springer, Berlin, Germany, 1985. 


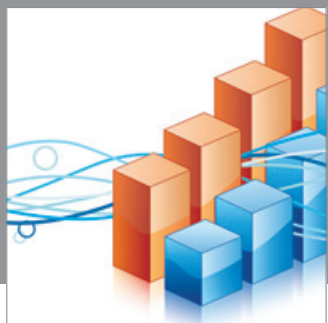

Advances in

Operations Research

mansans

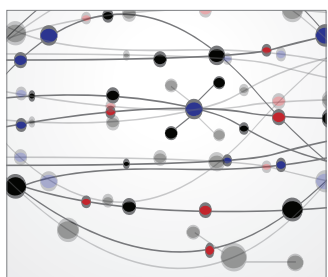

The Scientific World Journal
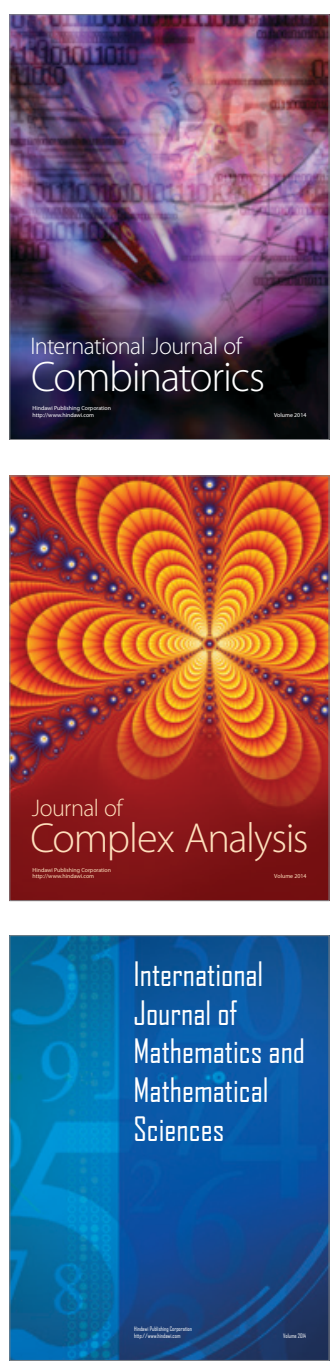
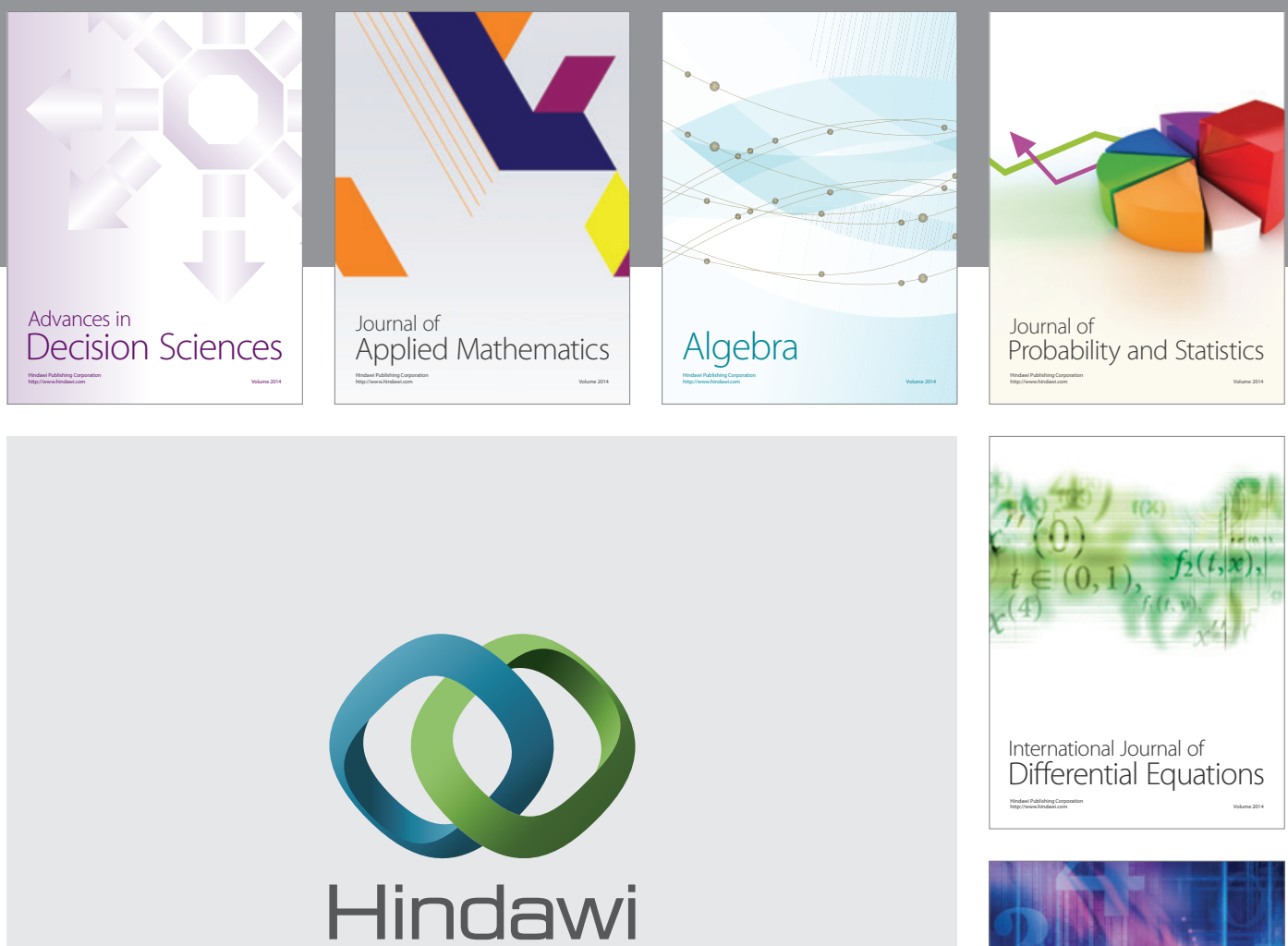

Submit your manuscripts at http://www.hindawi.com
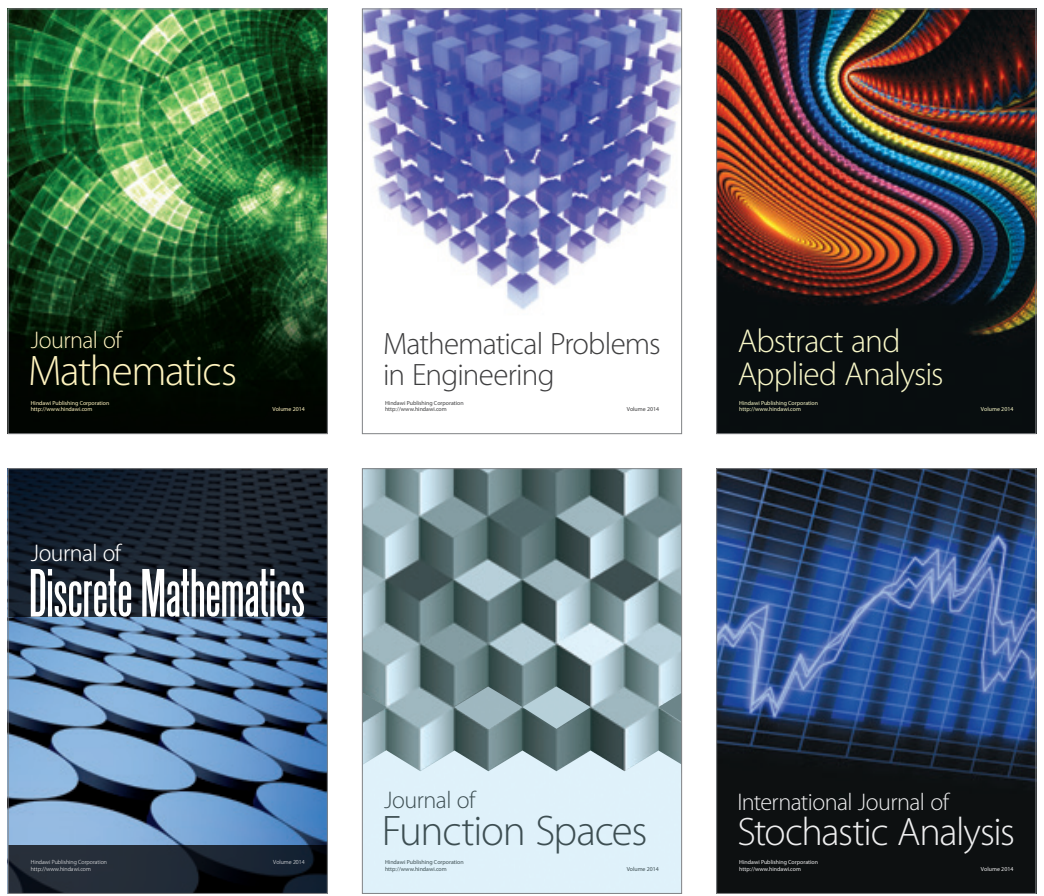

Journal of

Function Spaces

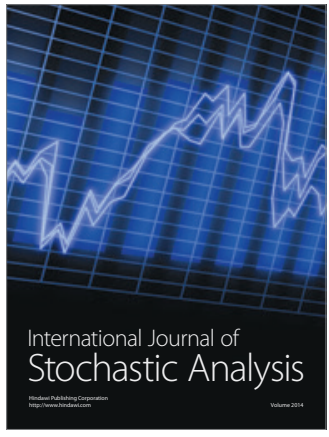

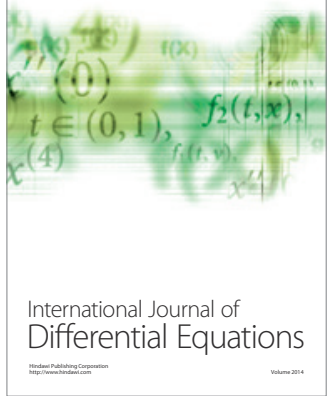
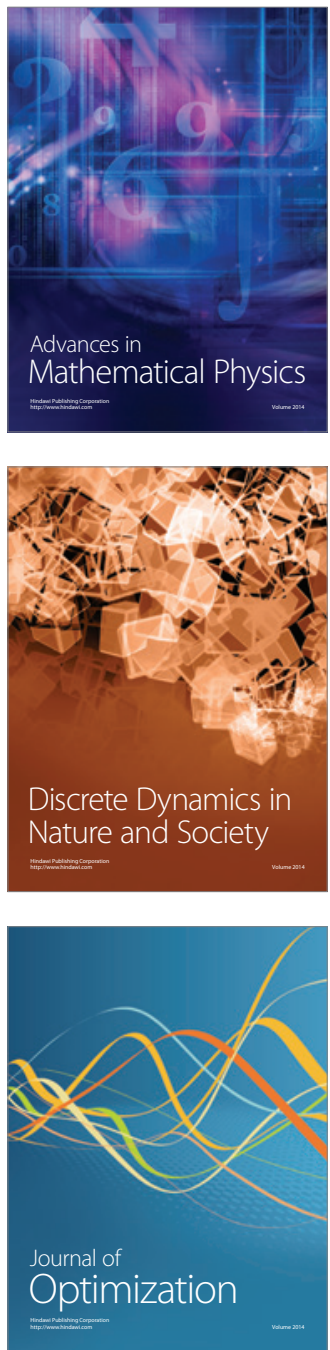